\title{
The origin of K-shell Emission of Neutral Iron Line from Sgr B2
}

\author{
D.O. Chernyshov ${ }^{* 1,3}$, V.A. Dogiel ${ }^{1}$, K. Koyama ${ }^{2}$, M. Nobukawa ${ }^{2}$, K.S. Cheng ${ }^{3}$ \\ ${ }^{1}$ I.E.Tamm Theoretical Physics Division of P.N.Lebedev Institute, Leninskii pr, 53, 119991 \\ Moscow, Russia \\ ${ }^{2}$ Department of Physics, Graduate school of Science, Kyoto University, Sakyo-ku, Kyoto 606-8502 \\ ${ }^{3}$ Department of Physics, University of Hong Kong, Pokfulam Road, Hong Kong, China
}

\begin{abstract}
We investigated the emission of neutral iron line from the massive molecular cloud Sgr B2. Despite the main part of the observed X-ray emission from molecular clouds is variable there is a possibility of presence of stationary component. The upper limit on the intensity of the stationary component is set by XMM-Newton observations of the Bridge complex. For Bridge complex the stationary component may be as high as $10 \%$ of the peak intensity of the X-Ray emission. We estimated the possible intensity of the stationary component expected from Sgr B2 in assumption that the stationary component is produced by subrelativistic protons. We made also predictions of evolution of the equivalent width of $6.4 \mathrm{keV}$ line from Sgr B2 expected in case of presence of the stationary component.
\end{abstract}

8th INTEGRAL Workshop: "The Restless Gamma-ray Universe"

September 27-30 2010

Dublin, Ireland

* Speaker. 


\section{Introduction}

The bright iron fluorescent $\mathrm{K} \alpha$ line emission from molecular clouds at the Galactic Center (GC) was predicted [ $[\mathbb{8}]$ and then discovered [ [5] for the cloud Sgr B2 more than twenty years ago. Later is was found from observations that the X-ray flux from Sgr B2 showed a steady decrease with time which is observed up to now [四, [0]]. This is a strong evidence that the origin of the variable component is a reflection of the primary X-Ray flare occurred several hundred years ago in the GC. The power of the source of primary X-rays is about $F_{f l} \sim 10^{39} \mathrm{erg} \mathrm{s}^{-1}$.

On the other hand, a number of observations indicated on even more energetic phenomena in the GC which cannot be caused by this flare. Thus, a hot plasma with the temperature about 10 $\mathrm{keV}$ was found in the GC which can be heated if there are sources with a power $\sim 10^{41} \mathrm{erg} \mathrm{s}^{-1}$ (see e.g. [[]]) which could be generated by energy release events in the past. In [[]] it was shown that this energy can be released if the central black hole captures a star. As a result, a flux of subrelativistic protons is ejected from the GC, which heats the central region with the necessary power. These protons can also produce $6.4 \mathrm{keV}$ line emission from molecular clouds which is, however, stationary because the lifetime of these protons $\tau_{p} \sim 10^{7} \mathrm{yr}$ [B] is much longer than the characteristic time of star capture by the central black hole $\left(\tau_{c} \sim 10^{5} \mathrm{yr}\right)$ [四].

However, observations of the 6.4 flux from Sgr B2 have not found up to now any evident stationary component, though recent analysis of continuum X-rays from the clouds observed by XMM-Newton (which are also supposed to be due to reflection of the primary X-rays too) might show this component $[\square]$. Thus, for the complex of molecular clouds Bridge one of the clouds shows rather stationary flux before a sudden one order of the magnitude jump of the intensity that was interpreted as a result of the X-front radiation which just had reached this cloud. Below we assume that this flux before the jump is produced by subrelativistic protons.

The stationary and time variable components of $6.4 \mathrm{keV}$ emission from the clouds have completely different origin. Therefore, we expect that derived parameters, such as an intensity of emission as well as an equivalent width of the line, may provide necessary information about processes producing X-ray emission from the clouds at the GC.

\section{Equivalent width}

In the framework of the reflection model the flux of continuum emission from a cloud is proportional roughly to

$$
F_{X} \propto n_{H} \sigma_{T} c N_{X}
$$

where $n_{H}$ if the hydrogen density in the cloud, $\sigma_{T}$ is the Thomson cross-section, and $N_{X}$ is the total number of primary photons with $E_{X} \sim 7.1 \mathrm{keV}$ inside the cloud.

The flux of $6.4 \mathrm{keV}$ line is

$$
F_{6.4} \propto n_{H} \sigma_{6.4} c \eta N_{X}
$$

where $\eta$ is the iron abundance in the cloud. Then the equivalent width of the line is

$$
e W=\frac{F_{6.4}^{X}}{F_{X}\left(E_{X}=6.4\right)} \propto \frac{\sigma_{6.4} \eta}{\sigma_{T}}
$$

that for the iron abundance $\eta=1.3$ gives $e W=1.13 \mathrm{keV}$ as observed for Sgr B2 [四]. 
The intensity of the $\mathrm{Fe} \mathrm{K} \alpha$ line excited by sub-relativistic particles (electrons or protons) in a cloud is

$$
F_{6.4}^{s r}=4 \pi \eta \int_{r} n_{H}(r) r^{2} d r \int_{E} d E \tilde{N}(r, E) v \sigma_{6.4}(E) .
$$

where $v$ and $E$ are the velocity and the kinetic energy of sub-relativistic particles, and $\sigma_{6.4}$ is the cross-section for $6.4 \mathrm{keV}$ line production by subrelativitic particles. The continuum bremsstrahlung emission can be calculated with the well-known cross-section of bremsstrahlung. One can find these cross-section in [0].

The expected equivalent width for power-law spectra of electron and protons $\left(N \propto E^{-\gamma}\right)$ and

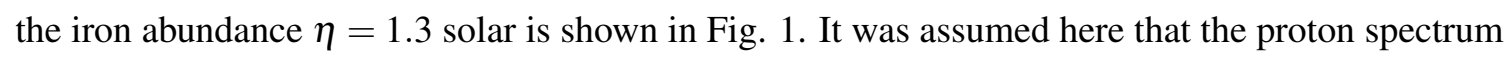
has a cut-off ( $N=0$ at $E>E_{i n j}$, see below).

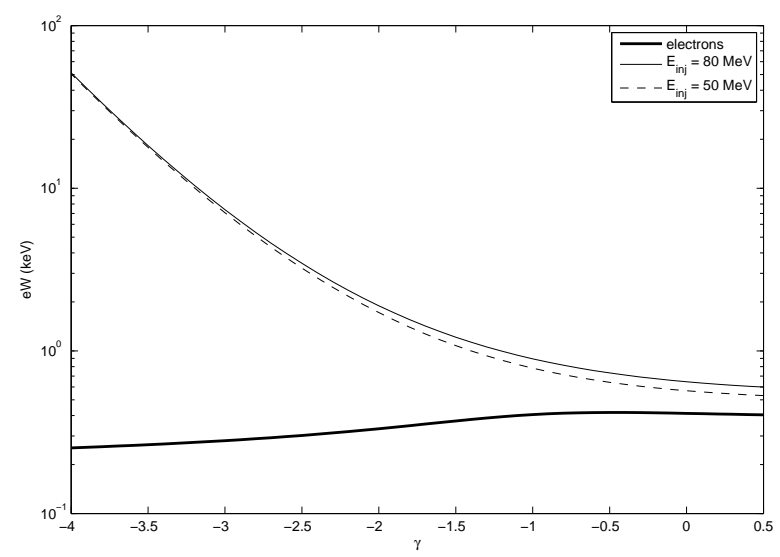

Figure 1: Equivalent width of $K_{\alpha}$ line for the solar abundance produced by electrons (thick solid line) and protons (thin solid line for injection energy $E_{i n j}=80 \mathrm{MeV}$, dashed line for injection energy $E_{i n j}=50 \mathrm{MeV}$ ) as a function of the spectral index $\gamma$.

One can see that the equivalent width of the $6.4 \mathrm{keV}$ line weakly depends on the spectral index of electrons while for the case of protons we expect strong variations of $e W$ with $\gamma$ value. Its value is much larger than that for the reflection process if the proton spectrum is soft.

\section{Stationary and time-variable components of X-ray emission from Sgr B2}

As it was shown in [2] when the central black hole captured a star, about $10^{57}$ subrelativistic protons were ejected with energies about $E_{p} \leq 100 \mathrm{MeV}$. The frequency of star capture is about $10^{-4}-10^{-5} \mathrm{yr}^{-1}$. The spatial and energy distribution of these protons in the central GC region can be calculated from the equation

$$
\frac{\partial N}{\partial t}-\nabla(D \nabla N)+\frac{\partial}{\partial E}\left(\frac{d E}{d t} N\right)=Q(E, t)
$$

where $d E / d t$ is the rate of Coulomb energy losses, $D$ is the spatial diffusion coefficient in the intercloud medium and the rhs term $Q$ describes the process proton injection in the GC. Because 
the lifetime of protons is longer than the characteristic time of star capture, the proton distribution is quasi-stationary and therefore the $\mathrm{X}$-ray emission from molecular clouds generate by these protons is quasi-stationary too.

With some modeling of proton penetration into the clouds (see for details [2]]) we can calculate the continuum and the $6.4 \mathrm{keV}$ line emission of clouds in the central region for any value of the diffusion coefficient $D$ which is unknown and therefore is a free parameter of the problem. For calculations we took parameters for the three clouds which are listed in [0]:

- Bridge, the density $n_{H}=1.9 \cdot 10^{4} \mathrm{~cm}^{-3}$, the radius of the cloud $r=1.6 \mathrm{pc}$, the distance from Sgr A* $R=63 \mathrm{pc}$;

- the same for for the cloud G0.11-0.11, $n_{H}=1.8 \cdot 10^{3} \mathrm{~cm}^{-3}, r=3.7 \mathrm{pc}, R=30 \mathrm{pc}$;

- the same for Sgr B2, $n_{H}=3.8 \cdot 10^{4} \mathrm{~cm}^{-3}, r=7 \mathrm{pc}, R=164 \mathrm{pc}$.

The density of photons of the primary X-ray flux from Sgr $\mathrm{A}^{*}$ decreases with the distance $R$ as: $\propto R^{-2}$. Then we can calculate for each of these clouds the ratio of the stationary component of the $6.4 \mathrm{keV}$ line produced by the protons $F_{6.4}^{p}$ to the time variable component at its peak value produced by primary X-rays $F_{6.4}^{X}$. To do this we use the observed ratio $F_{6.4}^{p} / F_{6.4}^{X}=0.1$ for the Bridge as it follows from the INTEGRAL data [ as a function of the diffusion coefficient $D$ as it is shown in Fig. 2 .

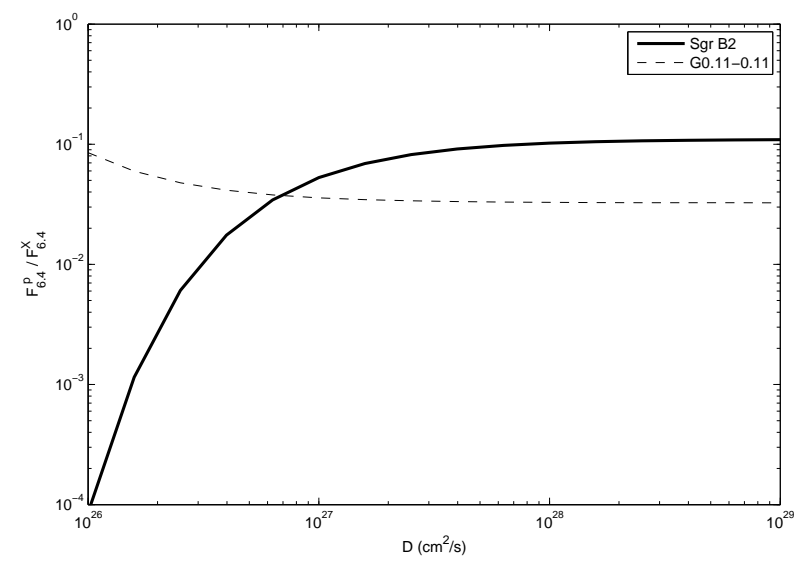

Figure 2: The ratio $F_{6.4}^{p} / F_{6.4}^{X}$ for the cloud G0.11-0.11 and Sgr B2 as a function of the diffusion coefficient $D$

One can see that protons can contribute to the total $6.4 \mathrm{keV}$ flux from Sgr B2 if the diffusion is large enough $D \geq 10^{27} \mathrm{~cm}^{2} \mathrm{~s}^{-1}$ then the expected stationary flux should be one order of the magnitude less than the observed $6.4 \mathrm{keV}$ emission from Sgr B2 near its maximum in 2000. For small values of $D$ there is no chance to observe $6.4 \mathrm{keV}$ emission after the $\mathrm{X}$-ray front has crossed the cloud. For the cloud G0.11-0.11 which is relatively close to Sgr $\mathrm{A}^{*}$ the situation is different. The intensity of stationary component is quite high almost independently of $D$. 


\section{Predicted variations of the Sgr B2 equivalent width in near future}

Observations show that the flux of the $6.4 \mathrm{keV}$ line emission from $\mathrm{Sgr} \mathrm{B} 2$ is rapidly decreasing with time (see the left panel of Fig. [3]). The question is whether we can find any evidence for a possible stationary component of Sgr B2. In Fig. B (right panel) we presented the expected variations of the Sgr B2 equivalent width when the flux generated by the primary X-rays $F_{6.4}^{X}$ is dropping down with the rate shown in the left panel of the figure. The calculations were done for different charge composition of subrelativistic particles (proton or electrons) and for different spectral index of these particles. One can see from the figure that if these particles are electrons the value of eW decreases and the rate of decrease are almost independent of the electron spectral index. In the case of protons the situation is intricate: for soft proton spectra the value of $e W$ should increase with time while for hard spectra it drops down.
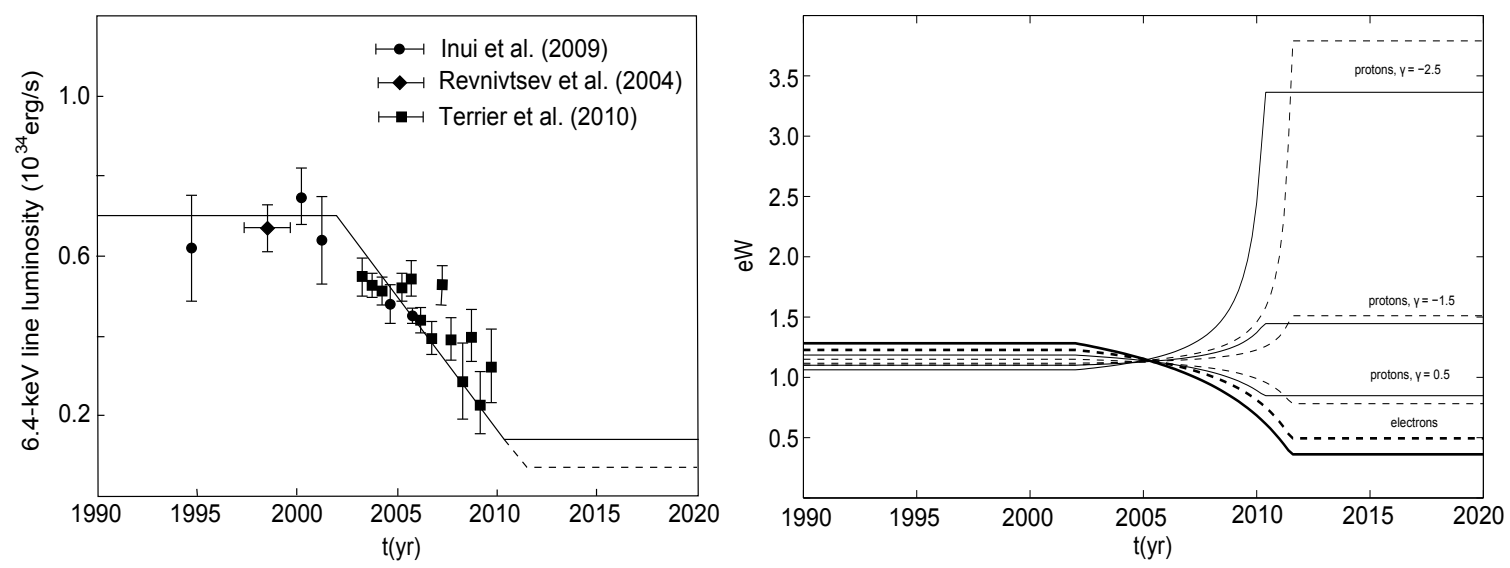

Figure 3: Left:The possible evolution of the Fe $\mathrm{K} \alpha$ line luminosity we used for our predictions. Right: The possible evolution of $\mathrm{Fe} \mathrm{K} \alpha$ line equivalent width. Dashed lines correspond to $F_{6.4}^{p} / F_{6.4}^{X}=0.1$, solid lines correspond to $F_{6.4}^{p} / F_{6.4}^{X}=0.2$.

\section{Acknowledgments}

DOC and VAD are partly supported by the RFBR grant 08-02-00170-a, the NSC-RFBR Joint Research Project RP09N04 and 09-02-92000-HHC-a. KSC is supported by a GRF grant of Hong Kong Government 7011/10p.

\section{References}

[1] Alexander, T. Stellar processes near the massive black hole in the Galactic center [review article], PhR, 419, (2005), 65

[2] Dogiel, V.A.; Cheng, K.-S.; Chernyshov, D.O.; Bamba, A.; Ichimura, A.; Inoue, H.; Ko, C.-M.; Kokubun, M.; Maeda, Y.; Mitsuda, K.; Yamasaki, N.Y., Origin of $6.4 \mathrm{keV}$ Line Emission from Molecular Clouds in the Galactic Center, PASJ, 61, (2009), 901 
[3] Dogiel, V. A., Tatischeff, V., Cheng, K.-S. et al. Nuclear interaction gamma-ray lines from the Galactic center region, $A \& A, \mathbf{5 0 8},(2009), 1$

[4] Inui, T.; Koyama, K.; Matsumoto, H.; Tsuru, T.G., Time Variability of the Neutral Iron Lines from the Sagittarius B2 Region and Its Implication of a Past Outburst of Sagittarius A, PASJ, 61, (2009), S241

[5] Koyama, K.; Maeda, Y.; Sonobe, T.; Takeshima, T.; Tanaka, Y.; Yamauchi, S., ASCA View of Our Galactic Center: Remains of Past Activities in X-Rays?, PASJ, 48, (1996), 249

[6] Koyama, K.; Hyodo, Y.; Inui, T. et al., Iron and Nickel Line Diagnostics for the Galactic Center Diffuse Emission, PASJ, 59, (2007), 245

[7] Ponti, G.; Terrier, R.; Goldwurm, A.; Belanger, G.; Trap, G. Discovery of a Superluminal Fe K Echo at the Galactic Center: The Glorious Past of Sgr A* Preserved by Molecular Clouds, ApJ, 714, (2010), 732

[8] Sunyaev, R. A.; Markevitch, M.; Pavlinsky, M. The center of the Galaxy in the recent past - A view from GRANAT, ApJ, 407, (1993), 606

[9] Tatischeff, V., X-and Gamma-Ray Line Emission Processes EAS Publications Series "Final Stages of Stellar Evolution" (Eds. C. Motch and J.-M. Hameury) 7 (2003) 79.

[10] Terrier, R.; Ponti, G.; Belanger, G.; Decourchelle, A.; Tatischeff, V.; Goldwurm, A.; Trap, G.; Morris, M. R.; Warwick, R., Fading Hard X-ray Emission from the Galactic Center Molecular Cloud Sgr B2, ApJ, 719, (2010), 143 